\title{
PERFORMANCE AND ROBUSTNESS ANALYSIS OF MODEL PREDICTIVE CONTROLLER (MPC) AND PROPORTIONAL INTEGRAL DERIVATIVE CONTROL (PID) OF AN AUTONOMOUS VEHICLE FOR LANE KEEPING MANEUVERS
}

\author{
Moveh Samuel \\ Department of Applied Mechanics and Design, School of Mechanical Engineering, Universiti Teknologi Malaysia, Malaysia81310 \\ UTM kudai johor Malaysia
}

\begin{abstract}
Motivated by Autonomous vehicle idea and driving safety issues, driver assistance system such as Active braking, Cruise Control, Lane departure warning lane keeping and etc. has become a very active research area. However, this paper presents the performance and robustness analysis of a model predictive control and proportional integral derivative control for lane keeping maneuvers of an autonomous vehicle using computer vision simulation studies. A simulation study was carried out where a vehicle model based on single tracked bicycle model was developed in MATLAB/SIMULINK environment together with a vision dynamic system. Both PID controller and MPC were simulated to maintain the desired reference trajectory of the vehicle by controlling steering angle. Further performance and robustness analysis were carried out and the simulation results show that the proposed control system for the PID control achieved its objective even though it was less robust in maintaining its performance under various conditions like vehicle load change, different longitudinal speed and different cornering stiffness. While in the case of MPC the optimizer made sure that the predicted future trajectory of the vehicle output tracks the desired reference trajectory and was more robust in maintaining its performance under same conditions as in PID.
\end{abstract}

Keyword: performance, robustness, analysis, lane keeping, maneuvers autonomous, vehicles

\section{Introduction}

With the current rate at which civilization and technological advancement is growing at a rapid rate, especially in the Automotive sector and in areas like development of auto-electronics technology. Active vehicular safety has been one of the most researched topic. Vehicle safety has attracted more and more attention in recent years especially in the automotive industry.

In a study of [1] Safety has become an important issue due to increasing number of vehicles and road users, resulting in the high rate of road accidents recorded every year. The same study also suggested that vast majority of these accidents are caused by driving errors, which can be avoided.

Car manufacturers are now focusing on developing new features to provide assistance to drivers, so as to improve safety and provide comfort. As stated in [2] Advance Driver Assistance System (ADAS) was developed to ensure safety and assistance to driver with the help of sensors 
technology and control algorithm, the system help to increase safety, adapt to surrounding and automate the car. The system have come a long way in a relatively short period of time, at least by the standards of the auto industry, which is well into its second century. The technology arguably has its roots in features that started showing up more than a decade ago, like adaptive cruise control, or perhaps even further back to the introduction of simple driver warning systems, such as emergency brake lights. According to [3] Some of the most common features that are in corperated in to the ADAS system includes: Collision avoidance system, Adaptive cruise control, automate braking, automate lighting, parking assist system, lane departure warning system and lane keeping system. This studies is focus more on one of such ADAS safety feature lane keeping.

The task of autonomously driving a car involve the following: lane detection, lane keeping, keeping safe distance in between vehicles, controlling speed of a vehicle according to traffic situation and road characteristics, lane change manuvers for overtaking vehicles, obstacle avoidance, establishing the shortest route to a destination, and parking within urban environments (Broggi et al 2004). It is a challenging task that has attracted the interest of researchers and research institutions globally. It is beieved that autonomously driven vehicles helps vehicles in keeping a short but reliable safe distance of $10 \mathrm{~m}-20 \mathrm{~m}$ thereby increasing roads and highways capacity. It helps to provide reduction in commuting time and autonomous driving leads to an optimal use of infrastructures. Remarkable fuel saving expenses is achieved by automatically controlling vehicles velocity as well as traffic decongestion (Sotelo et al, 2004).

In the automated driving mode of the autonomous car, the vehicle's lateral movement is controlled by a lateral control algorithm. The objective of this algorithm is to keep the vehicle in the center of it's lane. Position and orientation of the vehicle can be determined by the use of sensor such as camera used in identifying the white middle marker and left and right boundaries on the road and also by the use of sensors like Lidar for path tracking techniques both by explicitly or implicitly means. Regardless of the apparent simplicity of discovering white lane marks on road, it can be extremely hard to find white lane marks on different kind of road. These problems could be attributed to either of the following; shadows, occlusion by other vehicles, changes in the road surfaces itself, and different types of lane markings. A good lane detection algorithm should be capable of detecting all kind of lane marks from roadways and filter them to estimate an accurate estimate of the vehicle position relative to the lane.

\section{Control dynamics}

The lateral dynamics of vehicles has been discussed in [3], in which the kinematic and the dynamic models for lateral vehicle motion have been studied. Many control schemes have been established to control the lateral dynamics. The papers [4] and [5] have proposed control laws based on Proportional- Integral-Derivative (PID) control, while the paper [6] has developed a lateral controller based on fuzzy control. Other control technologies have also been used, such as Hœ control [7], [8], Linear Quadratic Regulator (LQR) [9] and sliding mode control [10], [11], [12]. In addition, the paper [13] has presented a feedback controller to control the lateral dynamics of rear-wheel drive cars subject to state constraints. However, these control methods are developed based on the linearized model of the lateral dynamics, while this paper studies the 
control problem for the nonlinear lateral dynamics. Model Predictive Control (MPC) is another method used in the trajectory tracking of autonomous cars, for example [14], [15], [16]. The computation time of nonlinear MPC is the main disadvantage of such an approach: this paper proposes an analytical solution to the lateral control problem which does not cause any computation burdens. Finally, the papers [17], [18] have studied the lateral control of independently actuated four wheeled vehicles. Comparisons of different lateral controllers can be found in [19], [20].

\section{Methodology}

The philosophy of the simulation work is always to use simple models, in order to understand more aspects possible about the physical system. A mathematical model was developed to predict the vehicle desired motion, stability and handling. The major response of the vehicle can be explained based on a linear vehicle model. The vehicle model used in this work was bicycle model, using an augmented version of two-degree-of-freedom (2DOF) elementary automobile as seen in figure 1 below. This model was linearized for simplicity and has consistently been proven in various studies [21], [22], [23], [24]. The nomenclatures used are presented in table 1

The lane keeping control was simulated for both PID control and MPC control in Matlab/Simulink environment under various conditions. Two different analyses were performed: performance and robustness analysis.

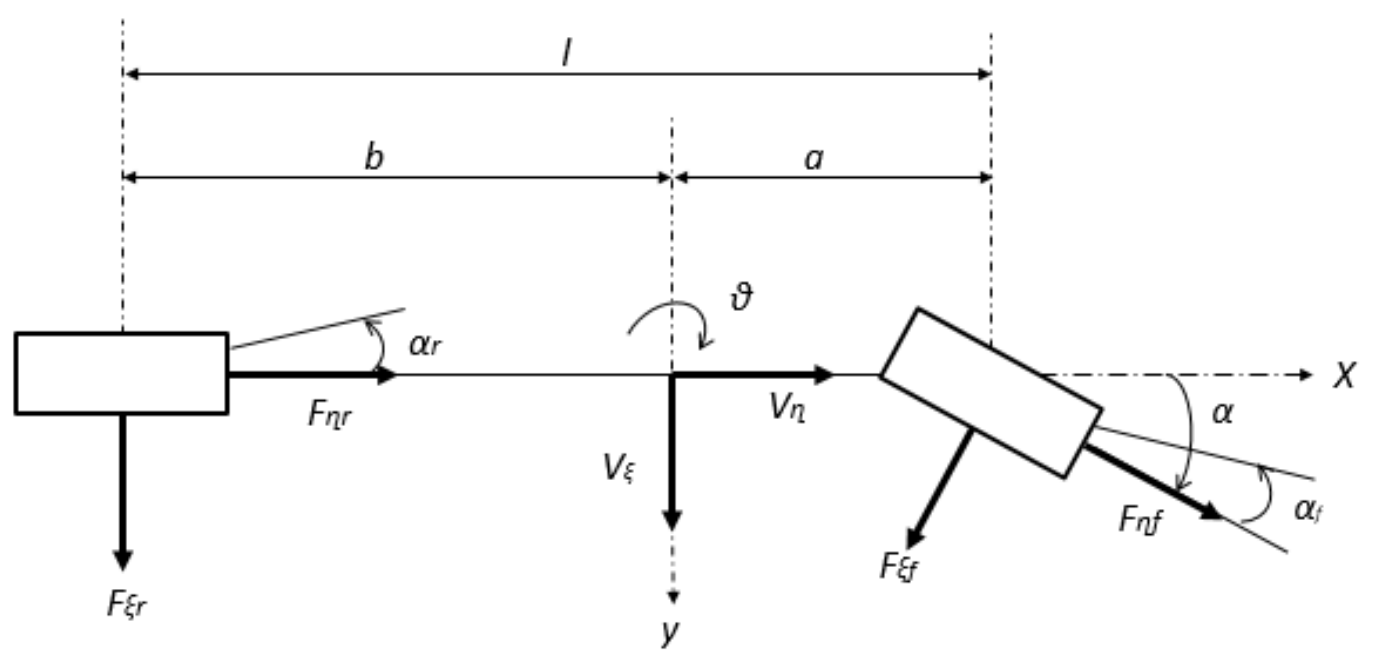

Figure 1: single track bicycle model 
Table 1:List of parameters for vehicle dynamic

\begin{tabular}{lll}
\hline $\mathbf{S} / \mathbf{N}$ & PARAMETER & SYMBOL \\
\hline 1 & front tire cornering stiffness & $\mathrm{C}_{\mathrm{af}}$ \\
2 & rear tire cornering stiffness & $\mathrm{C}_{\mathrm{ar}}$ \\
3 & vehicle longitudinal velocity & $\mathrm{V}_{\mathrm{x}}$ \\
4 & front tire distance & $\mathrm{a}$ \\
5 & rear tire distance & $\mathrm{b}$ \\
6 & motor inertia & $\mathrm{I}_{\mathrm{z}}$ \\
7 & mass of the vehicle & $\mathrm{m}$ \\
8 & front and rear side slip angle & $\mathrm{a}_{\mathrm{f}}, \mathrm{\alpha}_{\mathrm{r}}$ \\
9 & lateral tire force on front and rear tires $F \xi r, F \xi_{f}$ \\
10 & longitudinal/lateral velocity of vehicle in body reference frame \\
& \multicolumn{2}{c}{$V_{\xi}, V_{n}$} \\
\end{tabular}

\section{Results and Discussion}

\section{Performance and Robustness Analysis}

Performance analysis was be done by simulating the closed-loop system with longitudinal velocities of $\mathrm{Vx}=10,15,20,25 \mathrm{~m} / \mathrm{s}$ at a particular look-ahead distance $\mathrm{L}$ for both PID and MPC control using the same vehicle parameters in table 1. Robustness analysis for the PID and MPC closed-loop system were also done at different mass and cornering stiffness of the vehicle.

These simulations were carried out to test the performance and robustness of theses controller under various vehicle load, where the vehicle mass $m$ is varied by increasing the load and the cornering stiffness of the front wheel was varied to see the rubustness of the controls under different road conditions. All simulation results are shown below for both controls. 

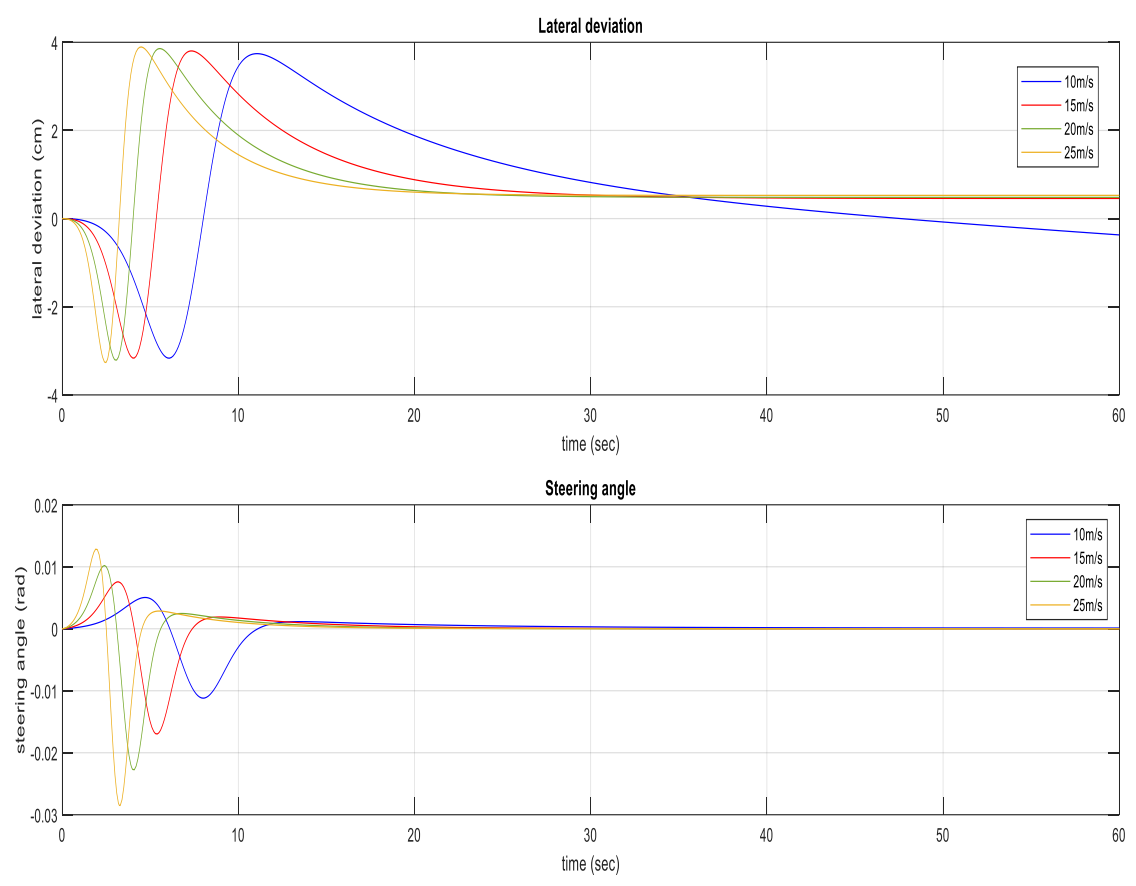

Figure 2: PID performance at different longitudinal velocity $V x$

From figure it was found that the controller was able to compensate the cross track error of the system by bringing it to zero as can be seen in the steering angle response. Although the response of the vehicle tends to overshoot at the beginning. This may cause passengers to be uncomfortable in a real scenario, before reducing the cross track error to zero. Also it can be observed that changing the longitudinal speed alters the performance. In this case the higher the speed the more the oscillations, but when the vehicle moves at a lower speed the oscillations and overshoot was less. As a conclusion, at a fixed look-ahead distance L, the speed should be varied from high too low for better performance even on a straight and curve roads. This is synonymous with the real situation while driving, especially at curved roads, where the speed is lowered for better cornering performance. 

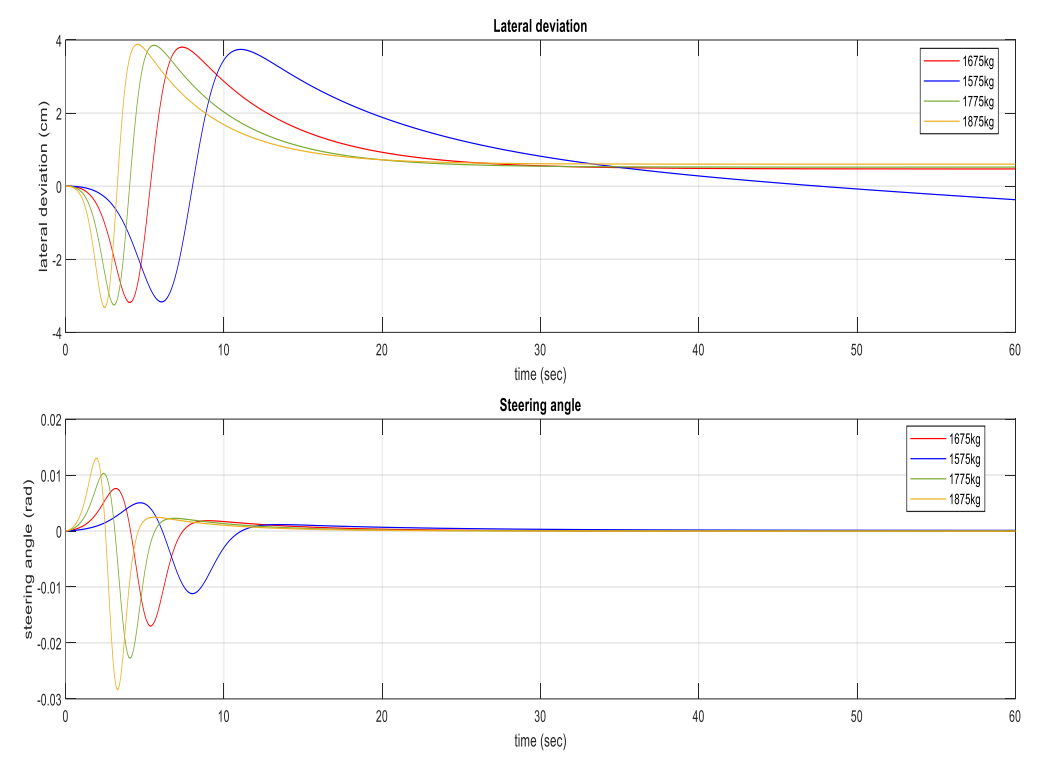

Figure 3: PID Roustness Analysis at different load (mass) Change

The first analysis was done for different values of the vehicle mass as can be seen in figure 3. This analysis was necessary to check how robust the vehicle will be under various vehicle load, as in the actual case of a real car with more than one passenger in it. This was achieved by simulating at different values of the vehicle mass $\mathrm{m}$. Also by changing the load capacity of the vehicle simulation results show that this affected the performance of the control. At low load capacity the vehicle was more stable, as it can be seen that the controller tries to steer the vehicle to its desire reference trajectory. But when the load capacity was increased, the response changed as can be seen in figure. At the highest load capacity the vehicle oscillation increased significantly as the controller tries to steer the vehicle to its desired reference trajectory 

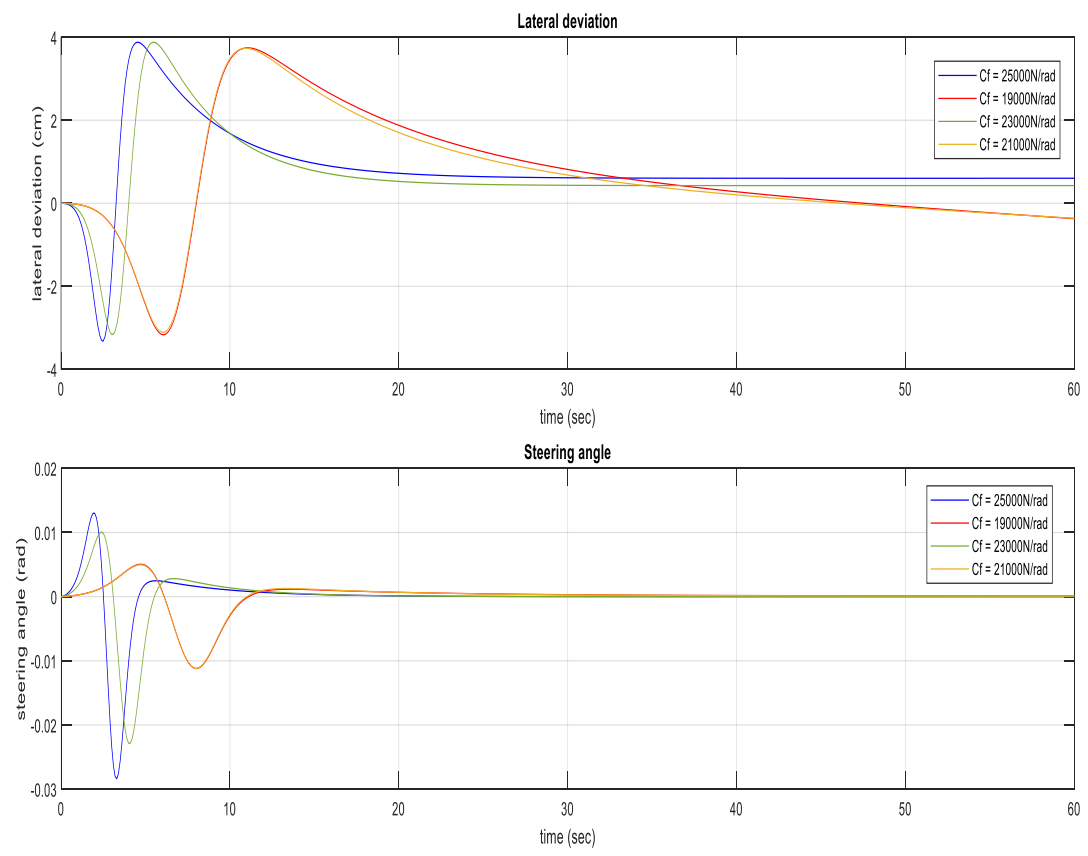

Figure 4: PID Roustness Analysis at different values of front cornering stiffness $\mathrm{C}_{\mathrm{f}}$

Also as seen in figure 4 the simulation of different value of front cornering stiffness was carried out also as in the case of driving the car in different road conditions. In the same manner and pattern, the controller's performance was altered when the front cornering stiffness was altered. When the simulation was carried out at a lower front cornering stiffness, the vehicle was able to follow the desired reference trajectory at a much quicker time as can be seen in figure 4 . But when the front cornering stiffness was increased, the vehicle behaviour changed. It can be seen that the vehicle had a wider oscillation before the controller was able to drive the vehicle to follow the desire reference trajectory as can be seen in figure. 

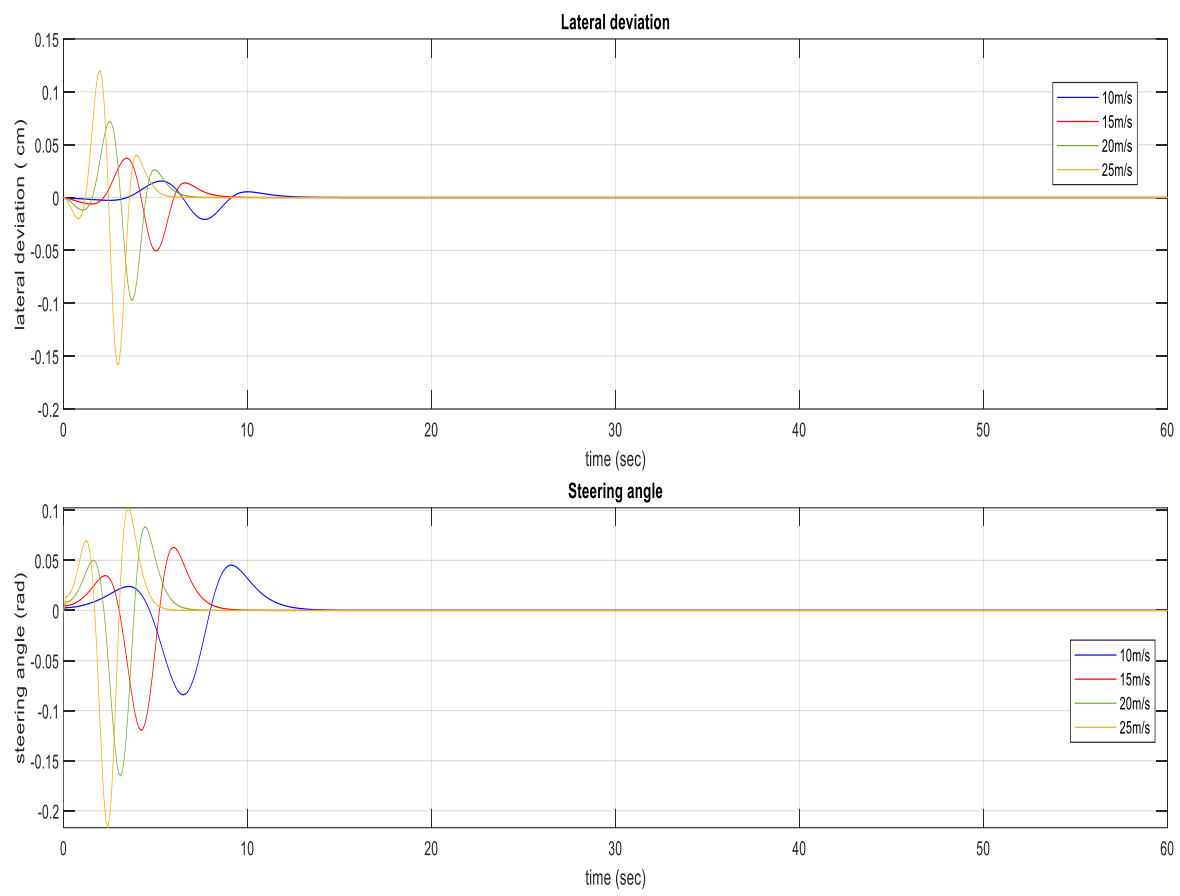

Figure 5: MPC performance at different longitudinal velocity Vx
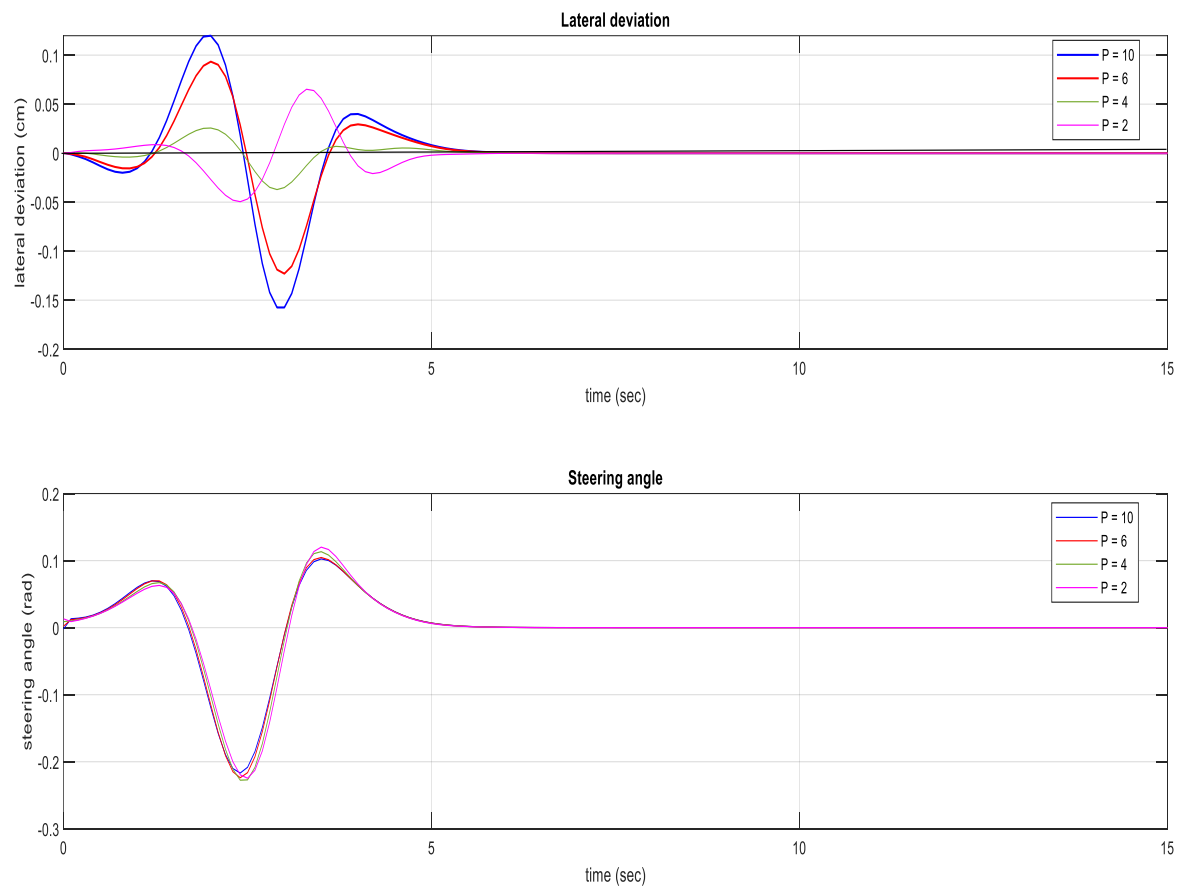

Figure 6: MPC response at different $\mathrm{P}$ time steps 
From figure, at the current time step, the MPC controller was solving the optimization problem over the prediction horizon while satisfying the constraints. The predicted path with the smallest $\mathrm{J}$ gives the optimal solution, and therefore determines the optimal steering wheel angle sequence that will get the vehicle as close as possible to the reference. At the current time step,

MPC applies only the first step of this optimal sequence to the vehicle and disregards the rest. Based on the applied steering wheel angle, the will travels some distance. At the next time step, the controller gets a new measurement of the vehicle's lateral position. It might be slightly different than what the MPC controller has predicted before. This could be due to some unmeasured disturbance acting on the vehicle. For example, it might be cast shadows or any other noise. Now the prediction horizon shifts forward by one time step and the controller repeats the same cycle of calculations to compute the optimal steering wheel angle for the next time step as seen in the figure. It should be noted that optimal performance of the MPC was achieved at a lower longitudinal speed, even though the MPC controller was still able to achieve its goal of adjusting the steering angle toward the desired reference trajectory and a higher longitudinal speed also.
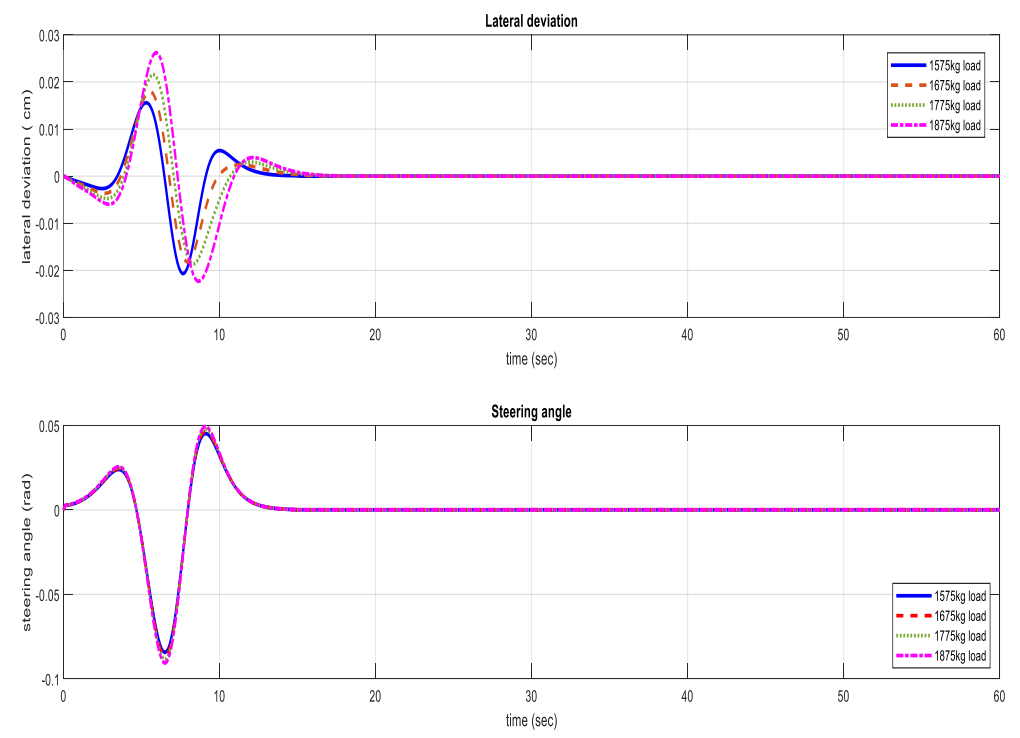

Figure 7 : MPC Roustness Analysis at different values of load Change (mass) 
From figure, it can be seen that simulating at various conditions of mass didn't really affect the MPC performance, although there was a little oscillation for the various masses, the optimizer was still able to solve the optimization problem and the controller tries to minimize the error between the reference and predicted path of the vehicle as seen in figure. This was achieved because of the characteristics of the MPC in using the vehicle model to predict the future trajectory of the vehicle. It also tries to minimize the change in the steering wheel angle from one time step to the next as seen in the simulation of the prediction horizon in figure 6 . The same thing can be seen in the simulation result at different front cornering stiffness in figure 4 . the different value of the front cornering stiffness did not really affect much on the performance of the MPC, because of the ability of the MPC in predicting the vehicle future for the next P time steps as stated earlier.
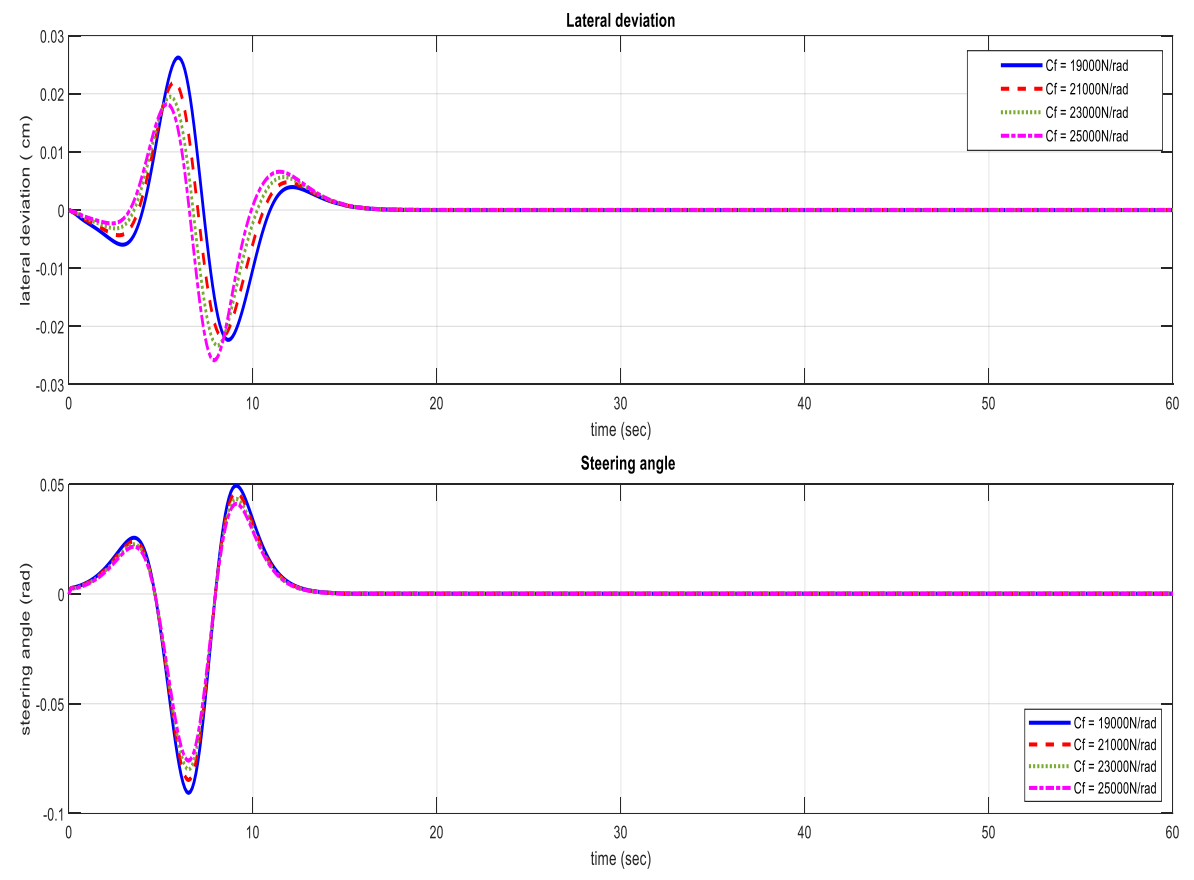

Figure 8: MPC Roburstness Analsis at different values of Front Cornering Stiffness

\section{Conclusion}

The task of autonomously driving a car isn't an easy task. In an automated driving mode of the autonomous car, the vehicle's lateral movement is controlled by a lateral control algorithm. The objective of this algorithm is to keep the vehicle in the center of its lane. Position and orientation 
of the vehicle can be determined by the use of sensor such as camera used in identifying the white middle marker and left and right boundaries on the road.

\section{Reference}

[1] M. Samuel, "A Review of some Pure-Pursuit based Path Tracking Techniques for Control of Autonomous Vehicle," vol. 135, no. 1, pp. 35-38, 2016.

[2] M. Syafiq and S. Mohamed, “ORIGINAL ARTICLE MALAYSIAN DRIVERS' PERCEPTION TOWARDS ADVANCED DRIVER ASSISTANCE SYSTEMS ( ADAS )," vol. 2, no. 2, pp. 15-20, 2017.

[3] S. A. Adnan Shaout, Dominic Colella, "Advanced Driver Assistance Systems - Past, Present and Future." 2011.

[4] H. Zhang and J. Wang, "Vehicle lateral dynamics control through afs/dyc and robust gainscheduling approach," IEEE Transactions on Vehicular Technology, vol. 65, no. 1, pp. 489-494, 2016.

[5] G. Han, W. Fu, W. Wang, and Z. Wu, "The lateral tracking control for the intelligent vehicle based on adaptive pid neural network," Sensors, vol. 17, no. 6, 2017.

[6] J. Yang and N. Zheng, "An expert fuzzy controller for vehicle lateral control," in Proc. of IEEE Annual Conference on Industrial Electronics, 2007, pp. 880-885.

[7] X. Huang, H. Zhang, G. Zhang, and J. Wang, "Robust weighted gainscheduling H $\infty$ vehicle lateral motion control with considerations of steering system backlash-type hysteresis," IEEE Transactions on Control Systems Technology, vol. 22, no. 5, pp. 1740$1753,2014$.

[8] C. Latrach, M. Kchaou, A. E. Hajjaji, and A. Rahbi, "Robust Hœ fuzzy networked control for vehicle lateral dynamics," in Proc. of IEEE Annual Conference on Intelligent Transportation Systems, 2013, pp. 905-910.

[9] R. Wang, Y. Sun, M. Lin, and H. Zhang, "Research on bus roll stability control based on LQR," in Proc. of International Conference on Intelligent Transportation, Big Data and Smart City, 2015, pp. 622-625.

[10] S. Lee and C. Chung, "Predictive control with sliding mode for autonomous driving vehicle lateral maneuvering," in Proc. of American Control Conference, 2017, pp. 29983003.

[11] G. Tagne, R. Talj, and A. Charara, "Higher-order sliding mode control for lateral dynamics of autonomous vehicles, with experimental validation," in Proc. of IEEE Intelligent Vehicles Symposium, 2013, pp. 678-683.

[12] C. Hatipoglu, U. Ozguner, and K. Redmill, "Automated lane change controller design," IEEE Transactions on Intelligent Transportation Systems, vol. 4, no. 1, pp. 13-22, 2003. 
[14] B. Gutjahr, L. Gr" oll, and M. Werling, "Lateral vehicle trajectory optimization using constrained linear time-varying MPC," IEEE Transactions on Intelligent Transportation Systems, vol. 18, no. 6, pp. 1586-1595, 2017.

[15] J. Levinson, J. Askeland, J. Becker, J. Dolson, D. Held, S. Kammel, J. Kolter, D. Langer, O. Pink, V. Pratt, M. Sokolsky, G. Stanek, D. Stavens, A. Teichman, M. Werling, and S. Thrun, "Higher-order sliding mode control for lateral dynamics of autonomous vehicles, with experimental validation," in Proc. of IEEE Intelligent Vehicles Symposium, 2011, pp. 163-168.

[16] P. Falcone, F. Borrelli, J. Asgari, H. Tseng, and D. Hrovat, "Predictive active steering control for autonomous vehicle systems," IEEE Transactions on Control Systems Technology, vol. 15, no. 3, pp. 566-580, 2007.

[17] J.Ahmadi,A.Sedigh,andM.Kabganian,“Adaptivevehiclelateral-plane motion control using optimal tire friction forces with saturation limits consideration," IEEE Transactions on Vehicular Technology, vol. 58, no. 8, pp. 4098-4107, 2009.

[18] J. Ni, J. Hu, and C. Xiang, "Envelope control for four-wheel independently actuated autonomous ground vehicle through AFS/DYC integrated control," IEEE Transactions on Vehicular Technology, vol. PP, no. 99, pp. 1-1, 2017.

[19] S.Dominguez,A.Ali,G.Garcia,andP.Martinet, “Comparisonoflateral controllers for autonomous vehicle: Experimental results," in Proc. of IEEE Conference on Intelligent Transportation Systems, 2016, pp. 1418-1423.

[20] G. Tagne, R. Talj, and A. Charara, "Design and comparison of robust nonlinear controllers for the lateral dynamics of intelligent vehicles," IEEE Transactions on Intelligent Transportation Systems, vol. 17, no. 3, pp. 796-809, 2016.

[21] M. Samuel, M. Mohamad, M. Hussein, and S. Saad, "Development of Lane keeping Controller Using Image processing," Int. J. Comput. Netw. Technol., vol. Volume 6, no. Issue 3, pp. 0-4, 2018.

[22] M. Samuel, M. Mohamad, S. M. Saad, and M. Hussein, "Development of Edge-Based Lane Detection Algorithm using Image Processing,” JOIV Int. J. Informatics Vis., vol. 2, no. 1, pp. 19-22, 2018.

[23] M. A. Hassan and M. Samuel, "Study Of Vehicle Handling Characteristics In Design Of Autonomous Vehicle For Sustainable Transportation,” vol. 4, no. 8, pp. 7857-7860, 2017.

[24] M. Samuel, "Comparative Analysis of Lane Detection Method Using Matlab," no. December, pp. 25-31, 2016. 